\section{Neutrinos of non-zero mass in Friedmann universes}

AN interesting comparison of independent methods for calculating the age of the Universe was proposed by Symbalisty et $a l .{ }^{1}$ to obtain a consistent age limit for the Universe in the range

$$
13.8 \mathrm{Gyr} \leqslant T_{\mathrm{U}} \leqslant 24 \mathrm{Gyr}
$$

This has prompted us to make some further applications by using these data to impose stringent limits on the mass of light $(\leqslant 1 \mathrm{MeV})$ neutrinos in Friedmann universes.

Cosmological arguments have been effectively ${ }^{2}$ used to set upper limits on neutrino masses using the values of the Hubble constant, $H_{0}$, and the deceleration parameter, $q_{0}$, in a universe uniformly populated with neutrinos. We obtain here such limits with the help of the data provided by Symbalisty et al. without any recourse to the values of the cosmological parameters $H_{0}, q_{0}$, which are in any case very uncertain.

In Friedmann models the present age $T_{U}$ of the Universe is given by ${ }^{3}$

$$
T_{\mathrm{U}}=H_{0}^{-1} f\left(q_{0}\right),
$$

where $f\left(q_{0}\right)$ is a monotonically decreasing function of $q_{0}$, attaining its maximum value at unity when $q_{0}$ approaches zero and tending to zero as $q_{0}$ becomes infinitely large. Now, Einstein's equations relate the measurable quantities $H_{0}, q_{0}$ and the present mass density $\rho_{0}$ of the Universe by the equation

$$
\rho_{0}=\frac{3 H_{0}^{2} q_{0}}{4 \pi G}
$$

This enables us to write

$$
T_{\mathrm{U}}=\left(\frac{3}{4 \pi G \rho_{0}}\right)^{1 / 2} q_{0}^{1 / 2} f\left(q_{0}\right)
$$

Let us now consider a situation at the present epoch where we can specify the mass density of the Universe and treat $q_{0}$ as a parameter. It can be easily demonstrated that the function $q_{0}^{1 / 2} f\left(q_{0}\right)$ increases monotonically, approaching a maximum of $\pi / 2^{3 / 2}$ as $q_{0}$ tends to infinity. Thus, the maximum possible age, $T_{\max }$, for closed Friedmann models $\left(q_{0}>\frac{1}{2}\right)$ is given by

$$
T_{\max }^{\text {closed }}=\pi\left(\frac{3}{32 \pi \mathrm{G} \rho_{0}}\right)^{1 / 2}
$$

and for open models $\left(0<q_{0}<\frac{1}{2}\right)$, we have

$$
T_{\max }^{\text {open }}=\left(\frac{1}{6 \pi \mathrm{G} \rho_{0}}\right)^{1 / 2}
$$

With the input of ages from Symbalisty et al. or from any considerations like nucleocosmochronometry, equations (5) and (6) can be used to obtain upper bounds on neutrino masses. Given the present age, $T_{\mathrm{U}}$, the requirement $T_{\mathrm{U}} \leqslant$ $T_{\max }$ provides maximum possible mass density $\rho_{0}$ for closed and open models. Let us consider neutrinos and antineutrinos of electron, muon and tau type, each having the same mass $m$. The present number density, $n_{\nu i}(0)$ of relic neutrinos and antineutrinos of type $i \cong 110 \mathrm{~cm}^{-3}$. Then for a typical age of $14 \times 10^{3} \mathrm{Myr}$ the upper bounds on the neutrino mass will be $38.70 \mathrm{eV}$ and $6.92 \mathrm{eV}$ for closed and open models, respectively. For the age of $20 \times$ $10^{3} \mathrm{Myr}$ the same numbers will be $18.91 \mathrm{eV}$ and $3.38 \mathrm{eV}$.

It is readily seen that the data on the age of the Universe place fairly tight limits on neutrino masses. The recent experimental limits ${ }^{4}$ give neutrino masses of the order of a few electron volts, although the Russian experiment ${ }^{5}$ reports them to be in the range $14-46 \mathrm{eV}$. Our results indicate that neutrino masses of the order of several tens of electron volts appear inconsistent with the lower limit on the age implied by nucleocosmochronometry. Again the experimental measurement of neutrino mass $\geqslant 14 \mathrm{eV}$ is not accommodated in standard open models. Thus, if the neutrinos were to have mass of a few tens of electron volts, the data on the age suggest that neutrinos would dominate the mass of the Universe, leading to its closure ${ }^{6,7}$ Further details and some more applications of these results will be published elsewhere ${ }^{8}$.

We thank J. V. Narlikar for discussions and D. Schram and E. M. D. Symbalisty for comments.

$$
\text { S. M. CHITRE† }
$$

Tata Institute of Fundamental

$$
\text { Research, }
$$

Homi Bhabha Road, Bombay 400005, India

* Present address: Department of Physics and Astronomy. University of Pittsburgh, Pittsburgh, Pennsylvania 15260 USA.

† Present address: Institute of Astronomy, Madingley Road Cambridge CB3 OHA, UK.

1. Symbalisty, E. M. D., Yang, J. \& Schramm, D. N. Nature 288, 143-45 (1980).

2. Cowsik, R. \& McClelland, J. Phys. Rev. Lett. 29, 669-70 (1972).

3. Weinberg, S. Gravitation and Cosmology (Wiley, New York, 1972).

4. Reines, F, Sobel, H. \& Pasierb, E. Phys. Rev, Lett. 45, 1307-1311 (1980)

Lubimov, V. A., Novikov, E. G., Nozik, V. Z., Tretyakov, E. F. \& Kosik, V. S. Phys. Lett. 94B, 266-268 (1980)

6. Zel'dovich, Ya B. \& Smorodinskii, Ya. A. Soviet Phys. JETP 14, 647-651 (1962).

. Schramm, D. N. \& Steigman, G. Astrophys. J. 243, 1-7 (1981).

8. Joshi, P. S. \& Chitre, S. M. Phys. Lett. A (submitted).

\section{Recent ${ }^{13} \mathrm{C} /{ }^{12} \mathrm{C}$ trends in atmospheric $\mathrm{CO}_{2}$ and tree rings}

FRANCEY's ${ }^{1}{ }^{13} \mathrm{C} /{ }^{12} \mathrm{C}$ record in tree rings from seven Tasmanian trees shows no systematic decrease. Such a decrease would be expected due to the dilution of the atmospheric $\mathrm{CO}_{2}$ pool by ${ }^{13} \mathrm{C} /{ }^{12} \mathrm{C}$ deficient $\mathrm{CO}_{2}$ released by fossil fuel combustion and forest clearing. Francey therefore questions the interpretation of ${ }^{13} \mathrm{C} /{ }^{12} \mathrm{C}$ tree ring records in terms of global $\mathrm{CO}_{2}$ behaviour.

Measurements of more than 30 trees from the Northern Hemisphere published elsewhere ${ }^{2-10}$ show, however, a systematic decrease of the ${ }^{13} \mathrm{C} /{ }^{12} \mathrm{C}$ ratio in tree rings over the second half of the past century (measurements of eight trees over this period) and the first quarter of this century, followed by some increase of the ratio between 1940 and 1960 . From about 1960 to 1975 a further decrease of the ${ }^{13} \mathrm{C} /{ }^{12} \mathrm{C}$ ratio is observed in our more extensive tree-ring record ${ }^{5,6}$ from measurements on 22 free-standing trees of different Northern Hemisphere origin (see Fig. 1). This decrease in mean ${ }^{13} \mathrm{C} /{ }^{12} \mathrm{C}$ tree-ring data compares favourably with the ${ }^{13} \mathrm{C} /{ }^{12} \mathrm{C}$ decrease in atmospheric $\mathrm{CO}_{2}$ between 1956 and 1978 determined directly by Keeling et al. ${ }^{11,12}$. Such a decrease is also found in measurements on Southern Hemisphere trees, for example one Brazilian tree determined by Rebello and Wagener ${ }^{13}$. Consequently, from a mean record calculation using all published tree-ring data I conclude that the ${ }^{13} \mathrm{C} /{ }^{12} \mathrm{C}$ ratio in modern wood has decreased significantly ${ }^{14}$. In fact, Pearman et al. ${ }^{15,16}$ obtained a ${ }^{13} \mathrm{C} /{ }^{12} \mathrm{C}$ trend on three Tasmanian trees consistent with trends of some other records, which they explained as climatic implications. These three trees are included in the seven trees used by Francey ${ }^{1}$ and now demonstrate the absence of any ${ }^{13} \mathrm{C} /{ }^{12} \mathrm{C}$ trend in tree rings.

Five of the Tasmanian trees were from a rain forest. Forest trees may not reflect the change in the ${ }^{13} \mathrm{C} /{ }^{12} \mathrm{C}$ ratio of atmospheric $\mathrm{CO}_{2}$ because of canopy effects and partial uptake of soil respiration $\mathrm{CO}_{2}$. The ${ }^{13} \mathrm{C} /{ }^{12} \mathrm{C}$ ratio in the latter would be

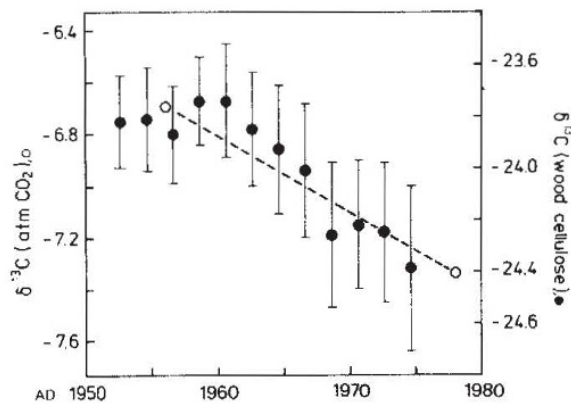

Fig. $1 \delta^{13} \mathrm{C}$ decrease in tree rings derived from about 22 trees of Northern Hemisphere origin for the $1952 / 53-1974 / 75$ period (O, right scale; adjustment of numerical data from Freyer ${ }^{6}$ to $\delta^{13} \mathrm{C}=-23.86 \%$ for average AD 1930-1950 wood cellulose) compared with the $\delta^{13} \mathrm{C}$ decrease in atmospheric $\mathrm{CO}_{2}$ observed by Keeling et al. ${ }^{11,12}(\mathrm{O}$, left scale). The two scales are offset by $-17.07 \%$ (from centre of gravity coordinates) to account for isotopic fractionation between atmospheric $\mathrm{CO}_{2}$ and the average of wood cellulose in these trees. The bars of tree-ring data represent $95 \%$ confidence limits based on Student's $t$ distribution. 